\title{
Electromagnetic Interference Shielding Effect of Nanocomposites with Carbon Nanotube and Shape Memory Polymer
}

\author{
Chun-Sheng Zhang ${ }^{\mathrm{a}}$, Qing-Qing $\mathrm{Ni}^{*}{ }^{\mathrm{b}}$, Shao-Yun $\mathrm{Fu}^{\mathrm{c}}$, and Ken Kurashiki ${ }^{\mathrm{a}}$ \\ ${ }^{a}$ Division of Advance Fibro-Science, Kyoto Institute of Technology \\ Matsugasaki sakyo-ku, Kyoto 606-8585, Japan \\ ${ }^{\mathrm{b}}$ Dept of Functional Machinery and Mechanics, Shinshu University \\ 3-15-1 Tokida, Ueda 386-8567, Japan \\ ${ }^{c}$ Technical Institute of Physics and Chemistry, Chinese Academy of Sciences (CAS) \\ Zhongguancun, Beijing 100080, China
}

* Corresponding author

Prof. Qing-Qing Ni

Dept of Functional Machinery and Mechanics, Shinshu University

3-15-1 Tokida, Ueda 386-8567, Japan

E-mail: niqq@shinshu-u.ac.jp

Fax: $+0081-268-215438$

Tel: $+0081-268-215438$ 


\begin{abstract}
The nanocomposites with carbon nanotubes (CNTs) and shape memory polymer (SMP) were developed for electrical applications. The specimens with different CNTs weight fractions were prepared. Their electrical resistivities and electromagnetic interference (EMI) shielding effectiveness (SE) were investigated. The electrical resistivity was examined by four-probe method at different testing temperatures of $25,35,45,55$ and $65{ }^{\circ} \mathrm{C}$ around glass transfer temperature $\left(\mathrm{T}_{\mathrm{g}}\right)$. As a result, for the developed nanocomposites, even lower weight fraction of CNTs could achieve a high level of conductivity and a low percolation threshold for CNTs content was confirmed. The electrical resistivity for the developed nanocomposites is dependant obviously on temperature with a linear relation like metals. The interconnected conducting network was formed more easily than other fillers. For the EMI SE measurements, a near-field antenna measurement system was used. The experiments to evaluate EMI SE were carried out in three different frequency bands, 8 26.5 $\mathrm{GHz}$ (K band), 33 50 GHz (Q band) and 50 75 GHz (V band). The EMI SE of CNT/SMP nanocomposites have a strong dependence of carbon nanotube content and the specimen thickness at all of three frequency bands. The higher frequency, the larger EMI SE. For the materials with $5.5 \mathrm{wt} \%$ VGCFs both experiment and analysis will agree well, and theoretical prediction proposed for EMI SE may be useful.
\end{abstract}

Key word: Carbon nanotube, Shape memory polymer, Electrical resistivity, Electromagnetic interference (EMI), Shielding effectiveness (SE) 


\section{Introduction}

In recent years, the progress of technology and increment in the amount of information are remarkable and high-speed communication is indispensable. To realize high-speed communication, the higher frequency range from the microwave to the millimeter wave is expected. Many electronic instruments with the higher frequency, such as satellite communication, automobile collision prevention radar, accident surveillance of a railroad, and millimeter wave wireless LAN and so on, have been developed and applied [1,2]. The electromagnetic waves produced from some electronic instruments have an adverse effect on the performance to other equipments. This is called as electromagnetic interference (EMI). EMI may cause malfunction to medical apparatus, industry robots or even cause harm to human body and become one of public nuisances [3-6]. Therefore, in order to alleviate these troubles the development of EMI shielding materials for microwave and millimeter waves are receiving increasing attention briskly.

EMI shielding refers to the reflection and/or adsorption of electromagnetic radiation by a material, which thereby acts as a shield against the penetration of the radiation through it [7]. The shield should be in high conductance, thus metals, such as steel, copper, aluminum, etc., are the most common materials used for EMI shielding. Since metal shielding has shortcomings of heavy weight, corrosion and physical rigidity, polymer composites with discontinuous conducting fillers, such as metal particles, carbon particles, carbon fiber, are extensively employed in EMI shielding [8-13]. Although these composites are not strong enough for most structural applications, they are attractive because of their superior molding and more dependable lightweight. For these composites, the EMI shielding effectiveness increases with increasing fraction of the filler and with increasing aspect ratio of the filler. The aspect ratio is defined as the ratio of the length to the diameter of the conductive fillers. According to the electromagnetic wave percolation theory, if the conductive filler in a polymer composite retains a high aspect ratio, the filler easily forms a conductive network. At a 
certain threshold value of fraction of the filler, the particles or fibers are sufficiently close-packed to form unbroken conducting pathway through the composite, and the conductivity of the material increases sharply $[10,14]$.

Among the various conductive fillers, carbon nanotubes (CNTs) have been shown to be an excellent candidate for EMI shielding due to higher aspect ratio and large surface area [14]. Vapor grown carbon nanofibers (VGCFs), referred as multiwall carbon nanotubes (CNTs), typically have diameters in the range of 50-200 $\mathrm{nm}$. Compared with other CNTs, VGCFs can be produced today in a large amount and at lower cost by using natural gas or coal as a stock catalyst $[15,16]$. Their good thermal and electrical conductivity, excellent mechanical properties, high aspect ratio (up to 1000) and low cost have attracted attention from both industrial and academic sides [17-19].

Many researches on the enhanced thermal, mechanical and electrical properties and EMI shield effect for VGCF base composites were conducted [15, 20-28]. However, the study on the unique nanocomposite with carbon nanotubes and shape memory polymer (SMP) is just on the way. The authors have reported the characteristics of shape memory effect on CNT/SMP nanocomposites in previous paper [29], where their electromagnetic interference (EMI) shielding effectiveness (SE) has not been investigated.

Shape memory polymer (SMP) has the characteristics such as large recoverability, lightweight, superior molding property and so on. These advantages have resulted in that the SMPs become one of functional materials from many fields [30-32]. For SMP of polyurethane series, its glass transfer temperature $\left(\mathrm{T}_{\mathrm{g}}\right)$ may be set up around room temperature, and it has a large difference in mechanical properties, optical characteristics and steam permeability above and below the temperature $\mathrm{Tg}$. The characterizations such as shape recovery and/or shape fixation may appear to be quite different. Thus the polyurethane SMP will have wider applications in the field of industry, medical treatment, welfare and daily life as an actuation material [33-36]. A significant property of 
SMP materials is their low stiffness. A few researchers have studied composite material based on a shape memory polymer matrix [37-39]. In our previous paper [29], carbon nanotube reinforced shape memory, CNT/SMP, nanocomposites were developed. The investigated results showed when a plate was made no matter how complicated shape was requested in the practical use, it could be obtained by heating and then molding the plate to a required one after cooling due to the shape stability of SMP. Moreover, any structure and its thickness in a product can be designed and controlled by laminating technology. Therefore, CNT/SMP nanocomposites provided that there exists sufficient intrinsic EMI shielding capability may be hopeful for electrical and electronic applications. Here, the electromagnetic interference (EMI) shielding effectiveness (SE) for the developed CNT/SMP nanocomposites are investigated and their electrical resistivities and the relationship between the electrical resistivity and temperature were examined. And EMI SE values for CNT/SMP nanocomposites with different thicknesses were compared.

\section{Experimental Work}

\subsection{Materials}

Multi wall carbon nanotubes (VGCFs) produced by Showa Denko K.K. were used with an average diameter of about $150 \mathrm{~nm}$ and the length of 10 20 $\mu \mathrm{m}$. The polyesterpolyol series of polyurethane shape memory polymer were used as a matrix and its glass transition temperature $\mathrm{T}_{\mathrm{g}}$ is about $45^{\circ} \mathrm{C}$. The raw material is liquid and the weight ratio of polymer to solvent is set to be 3:7.

\subsection{Fabrication of Specimens}

Carbon nanotubes were put into a solvent little by little and dispersed for $3 \mathrm{~h}$ at $45{ }^{\circ} \mathrm{C}$ by ultrasonic vibration with oscillation frequency of $42 \mathrm{kHz}$ and the power of $125 \mathrm{~W}$. The diluted SMP solution is gradually poured into the mixed solution of VGCFs and solvent, and then the mixture was dispersed for 3 hours. Then the mixture was cast into a container and dried at $70{ }^{\circ} \mathrm{C}$, and the 
CNT/SMP nanocomposites were prepared. In order to vaporize water completely, CNT/SMP nanocomposites were dried at $110{ }^{\circ} \mathrm{C}$. The weight fraction of VGCFs was $1.7,3.3,5.0$ and $6.7 \mathrm{wt} \%$, respectively. For comparison, the pure SMP films were also prepared in a similar method. The developed CNT/SMP nanocomposites were examined in a scanning electron microscope (SEM) in order to observe the distribution and structure of VGCFs in SMP. The SEM image of 5.0 wt $\%$ VGCFs is shown in Fig.1. It is observed that carbon nanotubes exhibited relatively good dispersion in SMP and were distributed randomly. It is confirmed that the size of carbon nannotubes in the material is $1 \sim 2$ hundred nanometers in diameter and several microns in length. It is evident that an interconnected conducting network has been formed in the developed CNT/SMP nanocomposites.

\subsection{Electrical resistivity}

In order to examine the electrical property of CNT/SMP nanocomposites, the electrical volume resistivity was conducted by four-probe method. A constant current was passed with a direct current (DC) voltage source (BP-3, Biocraft Co., Ltd.) through two outer electrodes and an output voltage was measured across the inner electrodes with the voltmeter (R6552, Advantest Co.). The specimen as shown in Fig.2 was cut from the nanocomposites sheet. Four copper electrodes with the size of $10 \times 5 \times 0.5 \mathrm{~mm}$ were affixed with electrically conductive adhesive (D-500, Fujikurakasei Co., Ltd.) to ensure good contact between the specimen surface and copper electrodes. The specimens with copper electrodes were then placed in a temperature-controlled chamber and heated for 5 minutes at the testing temperature. The volume resistivity measurements were performed under DC condition at $25,35,45,55$ and $65^{\circ} \mathrm{C}$, respectively. The electrical volume resistivity $\rho$ is calculated by Ohm's law:

$$
\rho=\frac{V}{I} \times \frac{w t}{l}
$$

where $V$ is voltage, $I$ is current, $l$ is distance between inside copper electrodes, $w, t$ is width and 
thickness of the specimen, respectively.

\subsection{Electromagnetic Interference (EMI) Shielding Effectiveness (SE)}

The methods such as free space method, shielding box method, coaxial transmission line method are often used to evaluate an electromagnetic shielding material. For a millimeter wave band, the technique to evaluate EMI SE is known widely with two antennas arranged face to face. A specimen much larger than the opening area of a horn antenna is required because of the radiation pattern characterization of the horn antenna. In this paper, the SE of CNT/SMP nanocomposites was analyzed using near-field antenna measurement systems. The illustration of measurement is shown in Fig.3, which consisted of a vector network analyzer (37169A Anritsu Co., Ltd.) and a near-field antenna measurement instrument (Tokai-techno Co., Ltd.) with a transmitting horn antenna and a receiving waveguide probe. The horn antenna and waveguide probe will be changed according to the frequency range to be measured.

The waveguide probe is perpendicularly arranged to the horn antenna and has the distance of more than three times of the wavelength from the opening side of the horn antenna for a measurement frequency. The output from the network analyzer is put into the horn antenna, and the receiving energy after transmitting CNT/SMP nanocomposites is measured by the waveguide probe. Based on these near-field data, the far-field value is computed by the network analyzer. In order to delete influence error of diffracted wave, only the transmitted electromagnetic wave is broached by using the time gating function of the network analyzer and considered to correspond to the transmitted energy. EMI SE was defined as the difference of the transmitted energy between the CNT/SMP nanocomposite specimen and a reference specimen. The SMP bulk is used as the reference specimen, and four kinds of CNT/SMP nanocomposites with different carbon nanotubes weight fractions are investigated. The EMI SE is measured in frequency ranges of $18 \sim 26.5 \mathrm{GHz}$ (K 
band), 33 50 GHz (Q band) and 50 75 GHz (V band) in the horn antenna and waveguide probe, respectively. In order to elucidate the influence of the specimen thickness on EMI SE, two different thicknesses, $0.5 \mathrm{~mm}$ and $3 \mathrm{~mm}$, are also prepared.

\section{Results and Discussion}

\subsection{Electrical resistivity results}

The electrical resistivities of CNT/SMP nanocomposites for different weight fractions at room temperature are shown in Fig.4. The electrical resistivity decreased with increasing the weight fractions of carbon nanotubes. It is just as expected the CNT/SMP nanocomposites possess electrical conductivity due to the addition of carbon nanotubes. The electrical resistivity of $1.7 \mathrm{wt} \%$ VGCFs is larger than others. For the weight fraction over $3.3 \mathrm{wt} \%$, the electrical resistivity dropped remarkably. This phenomenon is called as percolation. The big drop indicates the percolation threshold value of carbon nanotubes, VGCFs, in SMP for electrical resistivity is less than $3.3 \mathrm{wt} \%$. Shuying et al. [6] showed that the percolation threshold value for VGCF/liquid crystal polymer composites is $5.0 \mathrm{wt} \%$ VGCFs and Lozano [28] et al. showed that the percolation threshold value of VGCNF/Polypropylene composites is $18 \mathrm{wt} \%$ VGCFs. It is evident that for the matrix of shape memory polymer the VGCFs are dispersed to form an interconnected conducting network due to straight-chain molecules in polyurethane series of shape memory polymer used in this paper. It is known that the nanotubes content needed for percolation is strongly dependent on the ability to form an interconnected nanotube network. With the solvent and ultrasonic vibration for the dispersion of VGCFs in shape memory polymer, the VGCFs are distributed in SMP matrix uniformly and easily and resulted in low percolation threshold.

The electrical resistivities of CNT/SMP nanocomposites at different testing temperatures are shown in Fig.5. The electric resistivities became high with the rise of temperature. For CNT/SMP 
nanocomposites, the free electrons in VGCFs contribute electricity. The movement of these free electrons may be barred due to the molecules in a polymer matrix and this may cause resistance increase against a body. When the temperature arises and the movement of molecules becomes active, the collision frequency of free electrons increases and then electrical resistivity may become large. Therefore, for CNT/SMP nanocomposites, the electrical resistivity increased almost linearly with temperature like metal. If the electrical resistivity at $0{ }^{\circ} \mathrm{C}$ is set to be $\rho_{0}$ the electrical resistivity $\rho$ at temperature $T$ can be expressed as follows.

$$
\rho=\rho_{0}(1+\alpha T)
$$

where $\alpha$ is the temperature coefficient representing the rate of electrical resistivity.

\subsection{Prediction of EMI Shielding Effectiveness}

Electromagnetic wave consists of a magnetic field (H-field) and electric field (E-field) and changes periodically, while the two components induct each other and propagates at right angles to the plane containing two components perpendicular each other. The ratio of E-field to H-field is defined as wave impedance $\left(Z_{\mathrm{w}}\right.$, in ohms). Large impedance characterizes electric fields and small one is to magnetic fields. In the case of far field and plane wave conditions, the ratio of E-field to $\mathrm{H}$-field remains constant and equal to $120 \pi \Omega$, the intrinsic impedance of free space. In EMI shielding, there are two regions, the near field shielding region and far field shielding region, need to be considered. When the distance between the radiation source and the shield material is larger than $\lambda / 2 \pi$ (where $\lambda$ is the wavelength of the source wave), it is in the far field shielding region. The theory of electromagnetic plane wave is generally applied for EMI shielding in this region. When the distance is less than $\lambda / 2 \pi$, it is in the near field shielding region and the theory based on the contribution of electric and magnetic dipoles is used for EMI shielding $[5,12,40]$.

Shielding effectiveness, $\mathrm{SE}(\mathrm{dB})$, is a parameter for the reduction of EMI at a specific frequency 
due to a shield and is defined as: $[5,40]$

$$
S E=10 \log \frac{P_{0}}{P_{t}}=20 \log \frac{E_{0}}{E_{t}}=20 \log \frac{H_{0}}{H_{t}}
$$

where $P_{0}, E_{0}$ and $H_{0}$ are the energy, the electric and magnetic field intensity incident on the shield and $P_{t}, E_{t}$ and $H_{t}$ are the counterparts transmitted through the shield.

The EMI attenuation offered by a shield may depend on three mechanisms. The first is usually the reflection of the wave from the shield. The second is the absorption of the wave as it passes through the shield. The third is due to the re-reflections, i.e. the multiple reflections of the waves at various surfaces or interfaces within the shield. The reflection loss is a function of the ratio $\sigma_{r} / \mu_{r}$, whereas the absorption loss is a function of the product $\sigma_{r} \mu_{r}$, where $\sigma_{r}$ is the electrical conductivity of the shield relative to copper and $\mu_{r}$ is the magnetic permeability of the shield relative to free space. The multiple reflections, however, require the presence of large surface areas (e.g. a porous or foam material) or interface areas (e.g. a composite material containing fillers that have large surface area) in the shield. The loss connected with multiple reflections can be neglected when the distance between the reflecting surfaces or interfaces is large enough compared to the skin depth $[5,7]$, which in meters is defined:

$$
\delta=\frac{1}{\sqrt{\pi \mu \sigma}}
$$

where $f$ is the frequency (in Hz), $\mu$ is the magnetic permeability by $\mu=\mu_{0} \mu_{r}, \mu_{0}=4 \pi \times 10^{-7}$ $\mathrm{H} \mathrm{m}^{-1}$ is the absolute permeability of free space (air), and $\sigma$ is the electrical conductivity in $\Omega^{-1}$ $\mathrm{m}^{-1}$. For a VGCF/SMP nanocomposite, since it can be considered as a non-magnetic substance, we have $\mu_{r}=1$.

The shielding effectiveness (SE) for a conductive material can be expressed by the following equation.

$$
S E=R+A
$$


where $R$ is the first reflection loss of the electromagnetic wave, and $A$ is the absorption loss of the energy of the electromagnetic wave. According to the report by Morita et al. [41], when $t / \delta \geq 1.3$, the reflection loss $R(\mathrm{~dB})$ can be expressed as follows:

$$
R=20 \log \left(\frac{Z_{0} \delta \sigma}{2 \sqrt{2}}\right)
$$

And then the absorption loss, $A(\mathrm{~dB})$, can be evaluated from the equation as follows:

$$
A=8.686 \frac{t}{\delta}
$$

Therefore,

$$
S E=20 \log \left(\frac{Z_{0} \delta \sigma}{2 \sqrt{2}}\right)+8.68 \frac{t}{\delta}
$$

where, $Z_{0}=120 \pi, t$ is the thickness $(\mathrm{m})$ of a specimen . It is observed that SE in equation (8) is a function of resistivity, thickness and measurement frequency for a shied material.

When $t / \delta \leq 1.3$, SE will result in following formula.

$$
S E=20 \log \left(1+\frac{Z_{0} t \sigma}{2}\right)
$$

\subsection{Time Domain Property of Eelectromagnetic Wave}

The electromagnetic wave through a shield material is measured and then far-field values are computed using a network analyzer. Then these values are converted by inverse Fourier transform, and the time dependence of the transmitted electromagnetic wave is obtained. The typical relation between the time and transmitted electromagnetic waves is shown in Fig.6 at V-band for the specimen with $3 \mathrm{~mm}$ thickness. The transmitted electromagnetic wave is observed within $0.6 \sim 0.8 \mathrm{~ns}$ for CNT/SMP nanocomposites and SMP bulk. When VGCFs weight fraction increases, the peak of transmitted electromagnetic wave decreased and shifted to right side (delay). This means that EMI SE will increase with the increment of VGCFs weight fraction. Since the transmitted electromagnetic waves were only broached by the time gating function in the network analyzer they 
should correspond to the transmitted energy. The transmitted electromagnetic waves reached after $0.8 \mathrm{~ns}$ were the diffracted ones detouring around the specimen.

\subsection{Shielding Effectiveness of CNT/SMP Nanocomposites}

The EMI SE of CNT/SMP nanocomposites for different bands and shielding thicknesses is shown in Fig.7-9. For the variation of the thickness of a shielding material under the same frequency condition, the materials with $3 \mathrm{~mm}$ thickness show the higher EMI SE compared to the $0.5 \mathrm{~mm}$. According to the equation (7), it is clear that the absorption loss is in proportion relation to the thickness of a shielding material. When the thickness increases EMI SE will have a large value.

It is observed that EMI SE of CNT/SMP nanocomposites increased with VGCFs weight fraction at all frequency bands. The higher the frequency, the higher the value of EMI SE. For the material of $6.7 \mathrm{wt} \%$ VGCFs and $3 \mathrm{~mm}$ thickness, the value of EMI SE exceeded over $30 \mathrm{~dB}$ for any of three frequency bands, and its maximum value reached $65 \mathrm{~dB}$. The value of EMI SE indicates how much incident signal is blocked by the shielding medium. For the value of $10 \mathrm{~dB}$ EMI SE, this means $90 \%$ of incident signal is blocked, and for $20 \mathrm{~dB}, 99 \%$ of incident signal is blocked. Many researches indicate that the reduction of signal strength by $30 \mathrm{~dB}$ would be adequate for $50 \%$ cases in the applications of automotive and computer industries. And the reduction by $40 \mathrm{~dB}$ would fulfill $95 \%$ of the requirements [42]. It is clear that CNT/SMP nanocomposites are excellent shielding materials for electromagnetic interference, especially at high frequency.

For CNT/SMP nanocomposites for $6.7 \mathrm{wt} \%$ and $5.0 \mathrm{wt} \%$ of VGCFs with $3 \mathrm{~mm}$ thickness, the value of $t / \delta$ at maximal testing frequency $75 \mathrm{GHz}$ is 4.72 and 3.78 , respectively. Thus, using equation (8), the values of MEI SE for these two materials can be calculated. The relationships between MEI SE of CNT/SMP nanocomposites for $6.7 \mathrm{wt} \%$ and $5.0 \mathrm{wt} \%$ VGCFs with $3 \mathrm{~mm}$ thickness and the frequency at three bands are shown in Fig.10. In the testing frequency bands, the 
EMI SE for CNT/SMP nanocomposite with $6.7 \mathrm{wt} \%$ VGCFs is larger than that for $5.0 \mathrm{wt} \%$. When frequency becomes large the rate of increment for EMI SE in $6.7 \mathrm{wt} \%$ one is larger than that in 5.0 $\mathrm{wt} \%$. Making a comparison between experimental results and theoretical ones for CNT/SMP nanocomposite with $6.7 \mathrm{wt} \% \mathrm{VGCFs}$, the difference is $10 \mathrm{~dB}$ at $\mathrm{K}$ band, and about $20 \mathrm{~dB}$ at Q band and $\mathrm{V}$ band. The predicted value is smaller than experimental one. However, for CNT/SMP nanocomposite with $5.0 \mathrm{wt} \%$ VGCFs, no obvious difference at $\mathrm{K}$ band and only small difference of $8 \mathrm{~dB}$ at $\mathrm{Q}$ band and $\mathrm{V}$ band are observed. This difference may be contributed due to multiple reflection, which was not considered in the prediction.

\section{Conclusions}

In this study, the nanocomposites with carbon nanotubes (CNTs) and shape memory polymer (SMP) were developed and their electrical resistivity and electromagnetic interference shielding effectiveness (EMI SE) were examined. The results obtained are concluded as follows:

1. The electrical resistivity of CNT/SMP nanocomposites decreased with increasing the weight fraction of VGCFs at each testing temperature. The electrical resistivity for the developed nanocomposites is dependant obviously on temperature with a linear relation like metals. It is found the percolation threshold of VGCFs in shape memory polymer is about $10 \mathrm{wt} \%$.

2. The EMI SE of CNT/SMP nanocomposites have a strong dependence of carbon nanotube content and the specimen thickness at all of three frequency bands. The higher frequency, the larger EMI SE. The prediction for EMI SE is analyzed. For the materials with $5.5 \mathrm{wt} \%$ VGCFs both experiment and analysis will agree well, and theoretical prediction proposed for EMI SE may be respected for a practical use.

\section{Acknowledgments}


This work was supported by Kyoto Prefectural Technology Center for Small and Medium Enterprises. The author (Q.-Q. Ni) gratefully acknowledges the support of K. C. Wong Education Foundation, Hong Kong, and the key laboratory of Ministry of Education in Zhejiang Sci-Tech University, China and the CLUSTER of Ministry of Education, Culture, Sports, Science and Technology (MEXT), Japan.

\section{References}

[1] Hatakeyama K, Togawa H. Simplified measurement method of electromagnetic wave shielding and absorbing characteristics in mm-wave. IEICE Trans B-П 1998;J81-B-П:651-656.

[2] Harada Y. Research and development of frequency resources in MPT. J IEICE 1995;78:741-744.

[3] Das NC, Chaki Tk, Khastgir D, Chakraborty A. Electromagnetic interference shielding effectiveness of ethylene vinyl acetate based conductive composites containing carbon fillers. $\mathbf{J}$ Appl Polym Sci 2001;80:1601-1608.

[4] Chou K-S, Huang K-C, Shih Z-H. Effect of mixing process on electromagnetic interference shielding effectiveness of nickel/acrylonitrile-butadiene-styrene composites. J Appl Polym Sci 2005;97:128-135.

[5] Wang Y, Jing X. Intrinsically conducting polymers for electromagnetic interference shielding. Polym Adv Technol 2005;16:344-351.

[6] Yang S, Lozano K, Lomeli A, Foltz HD, Jones R. Electromagnetic interference shielding effectiveness of carbon nanofiber/LCP composites. Compos: Part A 2005;36:691-697.

[7] Chung DLL. Electromagnetic interference shielding effectiveness of carbon material. Carbon 2001;39:279-285.

[8] Shinagawa S, Kumagai Y, Urabe K. Conductive papers containing metallized polyester fibers for electromagnetic interference shielding. J Porous Mater 1999;6:185-190. 
[9] Heiser JA, King JA, Konell JP, Sutter LL. Shielding effectiveness of carbon-filled nylon 6,6. Polym Compos 2004;25:407-416.

[10] Lee BO, Woo WJ, Park HS, Hahm HS, Wu JP, Kim MS. Influence of aspect ratio and effect on EMI shielding of coating materials fabricated with carbon nanofiber/PVDF. J Mater Sci 2002;37:1839-1843.

[11] Das NC, Chaki TK, Khastgir D. Electromagnetic interference shielding effectiveness of conductive carbon black and carbon fiber-filled composites based on rubber and rubber blends. Adv Polym Techn 2001;20:226-236.

[12] Das NC, Khasgir D, Chaki TK, Chakraborty A. Electromagnetic interference shielding effectiveness of carbon black and carbon fiber filled EVA and NR based composites. Compos: Part A 2000;31:1069-1081.

[13] Luo X, Chung DDL. Electromagnetic interference shielding using continuous carbon-fiber carbon-matrix and polymer-matrix composites. Compos: Part B 1999;30:227-231.

[14] Ma C-C.M, Huang Y-L, Kuan H-C, Chiu Y-S. Preparation and electromagnetic interference shielding characteristics of novel carbon-nanotube/siloxane/poly (urea urethane) nanocomposites. J Polym Sci: Part B: Polym Phys 2005;43:345-358.

[15] Xu Y, Higgins B, Brittain WJ. Bottom-up synthesis of PS-CNT nanocomposites. Polym 2005;46:799-810.

[16] Rodriguez NM. A review of catalytically grown carbon nanofibers. J Mater Res $1993 ; 8: 3233-3250$.

[17] Heremans J, Beetz Jr. CP. Thermal conductivity and thermopower of vapor-grown graphite fibers. Phys Rev B 1985;32:1981-1986.

[18] Heremans J. Electrical conductivity of vapor-grown carbon fibers. Carbon 1985;23:431-436.

[19] Endo. M, Kim YA, Hayashi T, Nishimura K, Matusita T, Miyashita K, Dresselhaus MS. 
Vapor-grown carbon fibers (VGCFs): Basic properties and their battery applications. Carbon 2001;39:1287-1297.

[20] Patton RD, Pittman Jr. CU, Wang L, Hill JR, Day A. Ablation, mechanical and thermal conductivity properties of Vapor grown carbon fiber/phenolic matrix composites. Compos: Part A 2002;33:243-251.

[21] Patton RD, Pittman Jr. CU, Wang L, Hill JR. Vapor grown carbon fiber composites with epoxy and poly(phenylene sulfide) matrices. Compos: Part A 1999;30:1081-1091.

[22] Richard P, Prasse T, Cavaille Y, Chazeau L, Gauthier C, Duchet J. Reinforcement of rubbery epoxy by carbon nanofibres. Mater Sci Eng A 2003;352:344-348.

[23] Carneiro OS, Covas JA, Bernardo CA, Caldeira G, Van Hattum FWJ, Ting JM, Alig RL, Lake ML. Production and assessment of polycarbonate composites reinforced with vapour-grown carbon fibres. Compos Sci Technol 1998;58:401-407.

[24] Chen Y-M, Ting J-M. Ultra high thermal conductivity polymer composites. Carbon 2002;40:359-362.

[25] Takahashi T, Yonetake K, Koyama K, Kikuchi T. Polycarbonate crystallization by vapor-grown carbon fiber with and without magnetic field. Macromol. Rapid Commun. $2003 ; 24: 763-767$.

[26] Kuriger RJ, Alam MK, Anderson DP, Jacobsen RL. Processing and characterization of aligned vapor-grown carbon fiber reinforced polypropylene. Compos: Part A 2002;33:53-62.

[27] Lozano K, Barrera EV. Nanofiber-reinforced thermoplastic composites. I. thermoanalytical and mechanical analyses. J Appl Polym Sci 2001;79:125-133.

[28] Lozano K, Bonilla-Rios J, Barrera EV. A study on nanofiber-reinforced thermoplastic composites (П): investigation of the mixing rheology and conduction properties. J Appl Polym Sci 2001;80:1162-1172. 
[29] Compos Struct. To be Online.

[30] Irie M. Development of shape memory polymers. CMC, 2000.

[31] Lendlein A, Kelch S. Shape-memory polymers. Angew Chem Int Ed 2002;41:2034-2057.

[32] Tobushi H, Okumura K, Endo M, Hayashi S. Thermomechanical properties polyurethane-shape memory polymer form. J Intell Mater Syst Struct 2001;12:283-287.

[33] Tobushi H, Hayahi S, Ikai A, Hara H. Basic deformation properties of a polyurethane-series shape memory polymer film. Trans Jpn Soc Mech Eng A 1996; 62(594):576-582.

[34] Jeong HM, Lees JB, Lee Y, Kim BK. Shape memory polyurethane containing mesogenic moiety, J Mater Sci 2000;35;279-283.

[35] Lin JR, Chen LW. Study on shape-memory behavior of polyether-based polyurethanes. I. Influence of the hard-segment content. J Appl Polym Sci 1998;69:1563-1574.

[36] Lin JR, Chen LW. Study on shape-memory behavior of polyether-based polyurethanes. II. Influence of the soft-segment molecular weight. J Appl Polym Sci 1998;69:1575-1586.

[37] Ohki Ta, Ni Q-Q, Ohsako N, Iwamoto M. Mechanical and shape memory behavior of composites with shape memory polymer. Compos: Part A 2004;35:1065-1073.

[38] Gall K, Dunn ML, Liu Y, Finch D, Lake M, Munshi NA. Shape memory polymer nanocomposites. Acta Materialia 2002;50:5115-5126.

[39] Liang C, Rogers CA, Malafeew E. Investigation of shape memory polymers and their hybrid composites. J Intell Mater Syst Struct 1997;8:380-386.

[40] Lu G, Li X, Jiang H. Electrical and shielding properties of ABS resin filled with nickel-coated carbon fibers. Comp Sci Technol 1996;56:193-200.

[41] Morita T, Sumihiro S, Okumura K. Measuring the electro magnetic shielding effectiveness of materials using the metal spray coating technique. IEICE Technical Report. Electromagnetic Compatibility 2000;99:19-24. 
[42] Simon RM. EMI shielding through conductive plastics. Polym Plast Technol Eng 1981; 17(1):1-10 


\section{The List of captions for Figures}

Fig.1 SEM micrograph of CNT/SMP nanocomposite with $5.0 \mathrm{wt} \%$ VGCFs.

Fig.2 Specimen used for measurement of electrical resistivity by four-probe method.

Fig.3 The schematic of an EMI shielding effectiveness measurement system.

Fig.4 The electrical resistivites of CNT/SMP nanocomposites by four-probe method

Fig.5 The relationships between electrical resistivites and temperature for different CNT/SMP nanocomposites

Fig.6 The transmitted electromagnetic wave in a time domain.

Fig.7 The shielding effectiveness in K-band frequency range for CNT/SMP nanocomposites with different thicknesses, (a) $3 \mathrm{~mm}$, (b) $0.5 \mathrm{~mm}$.

Fig.8 The shielding effectiveness in Q-band frequency range for CNT/SMP nanocomposites with different thicknesses, (a) $3 \mathrm{~mm}$, (b) $0.5 \mathrm{~mm}$.

Fig.9 The shielding effectiveness in V-band frequency range for CNT/SMP nanocomposites with different thicknesses, (a) $3 \mathrm{~mm}$, (b) $0.5 \mathrm{~mm}$.

Fig.10 Theoretical prediction of shielding effectiveness for CNT/SMP nanocomposites with $3 \mathrm{~mm}$ thickness, (a) K-band, (b) Q-band, (c) V-band. 


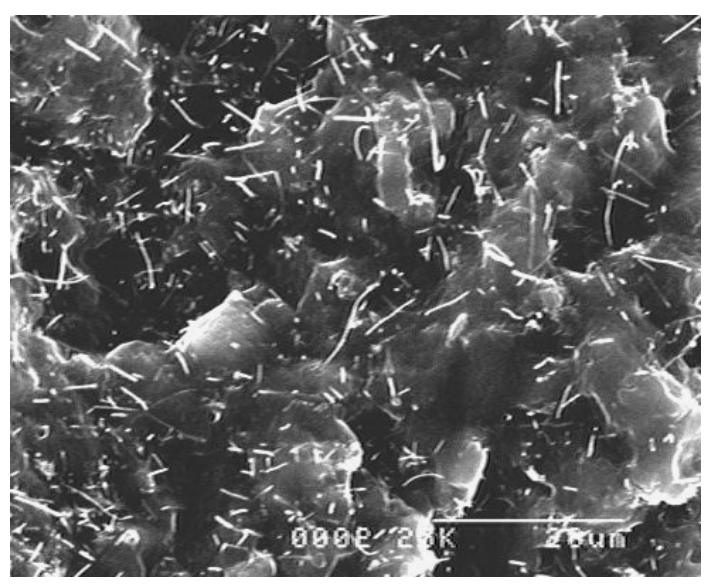

Fig.1 SEM micrograph of $5.0 \mathrm{wt} \% \mathrm{CNT} / \mathrm{SMP}$ nanocomposites

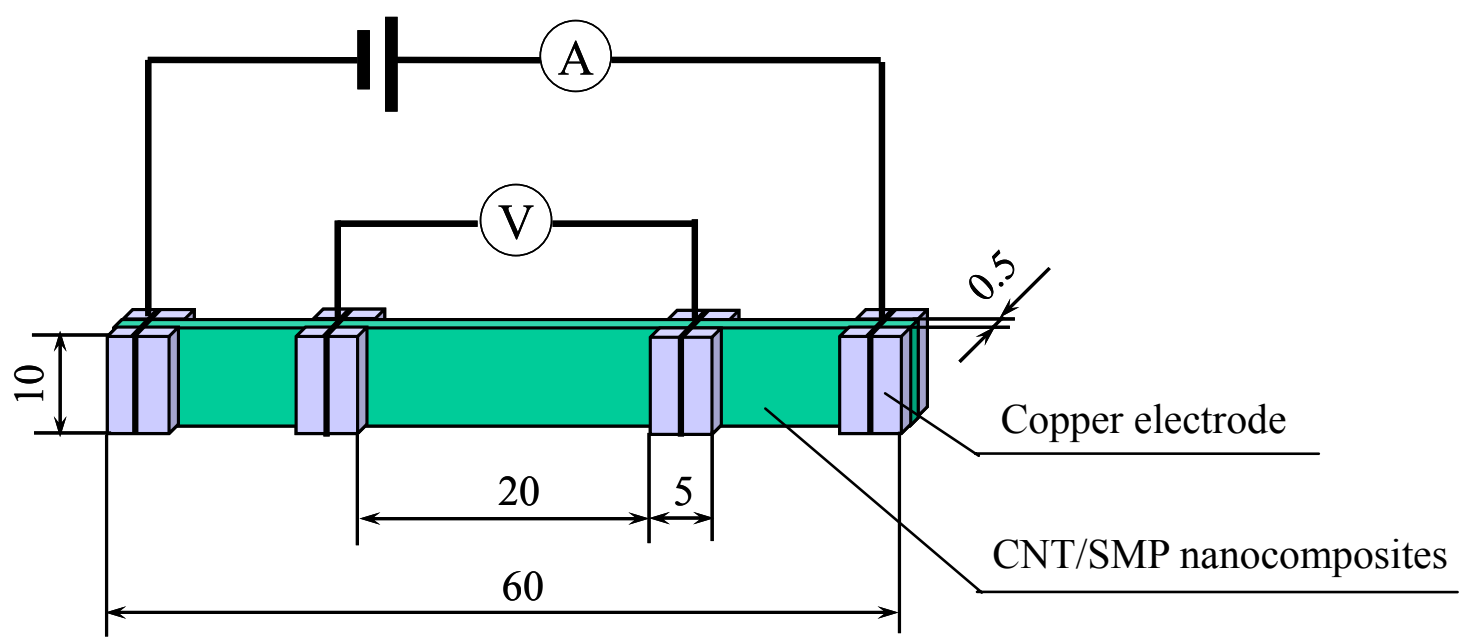

Fig.2 Specimen used for measurement of the electrical resistivites by four-probe method 


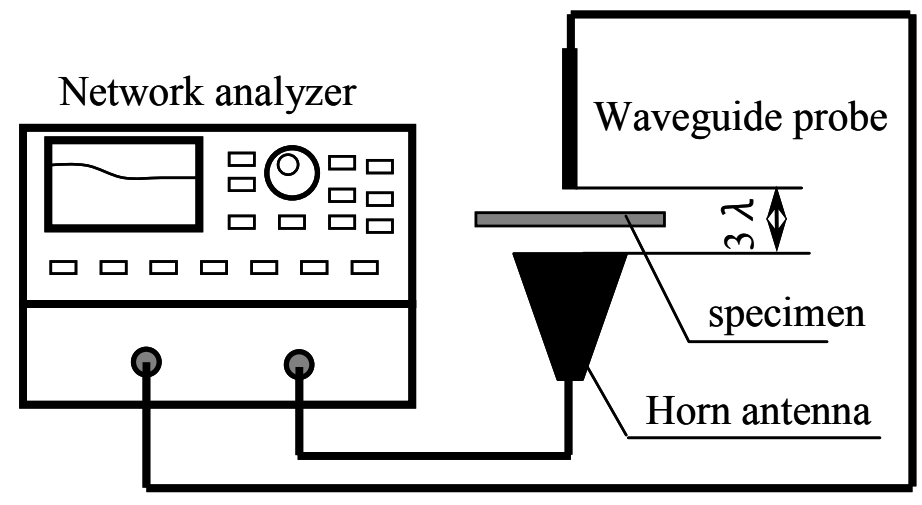

Fig.3 The schematic of an EMI shielding effectiveness measurement system 


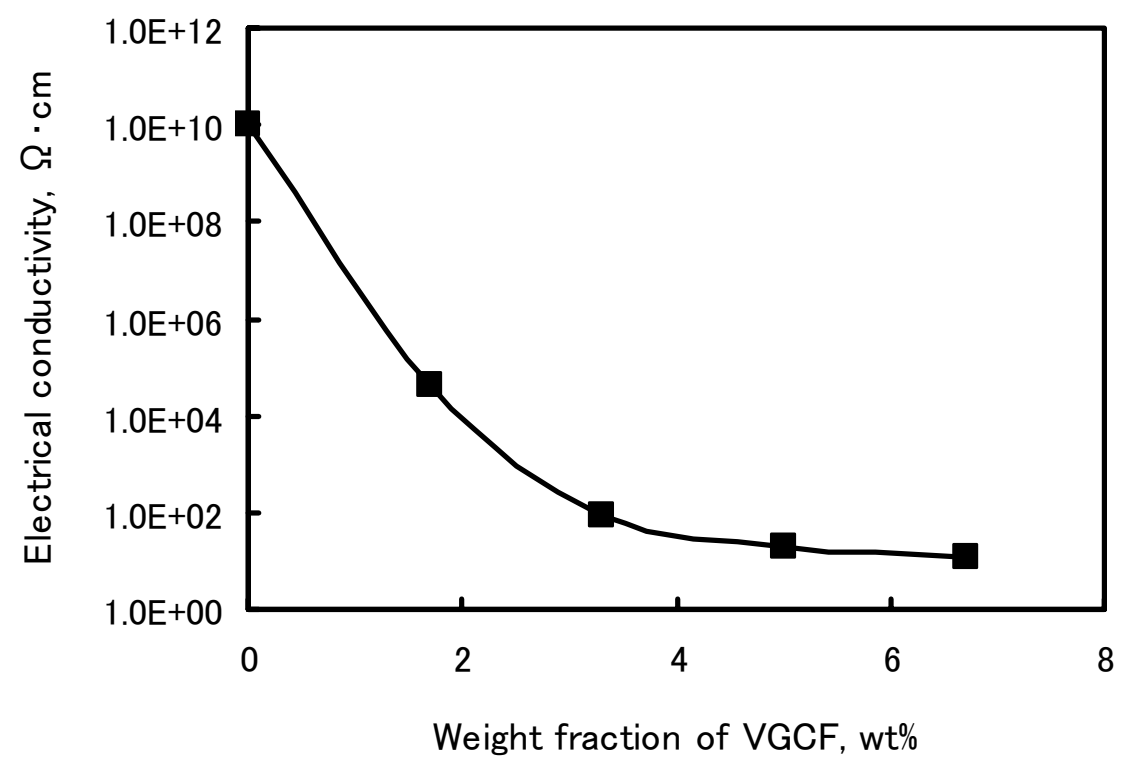

Fig.4 The electrical resistivites of CNT/SMP nanocomposites from four-probe method

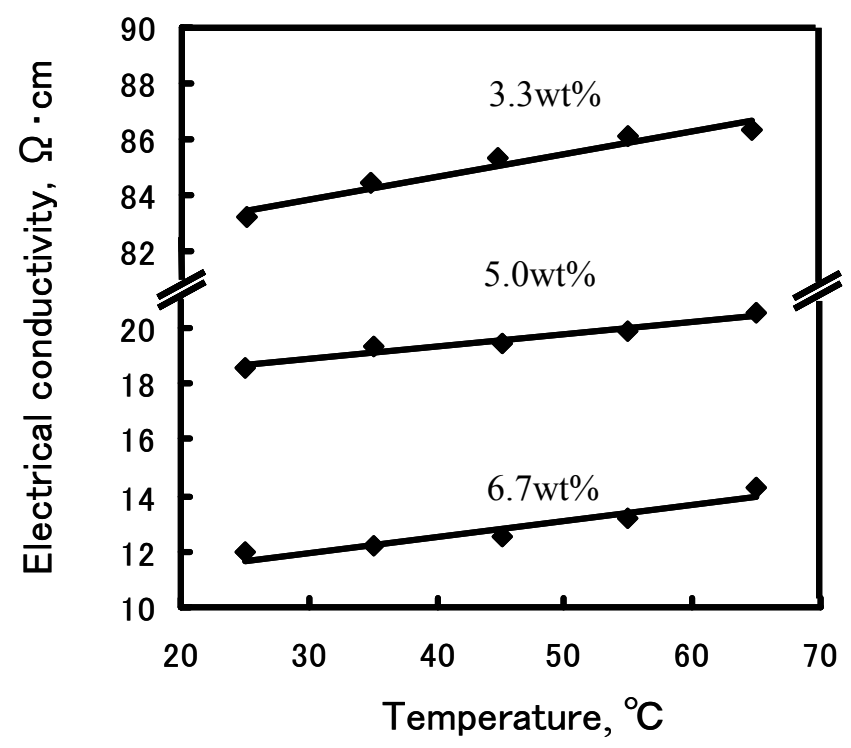

Fig.5 The relationship between electrical resistivites of CNT/SMP nanocomposites and temperature 


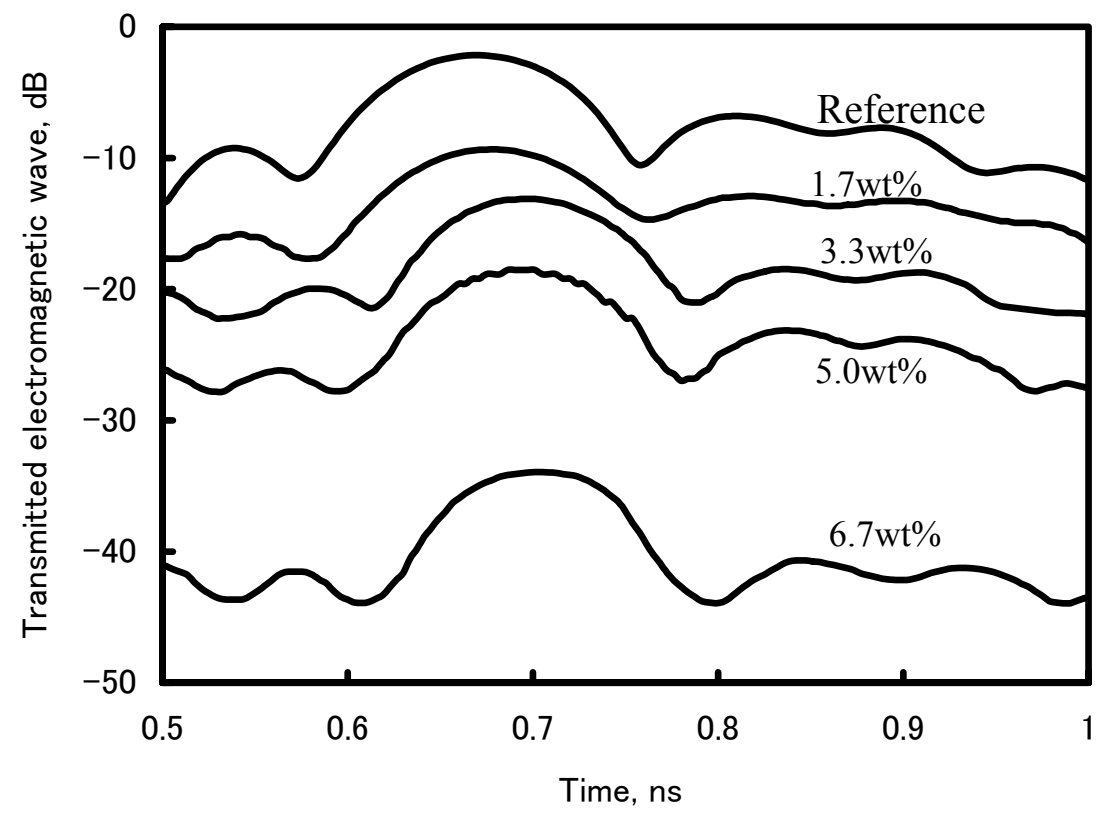

Fig.6 The time domain of the transmitted electromagnetic wave 


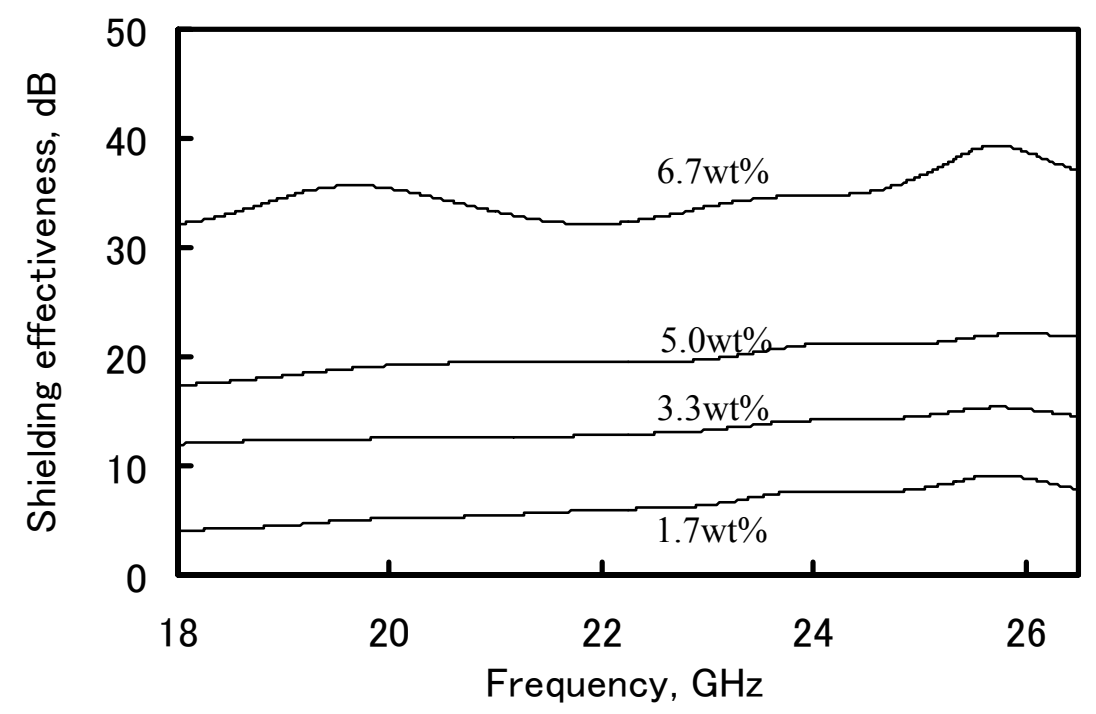

(a)

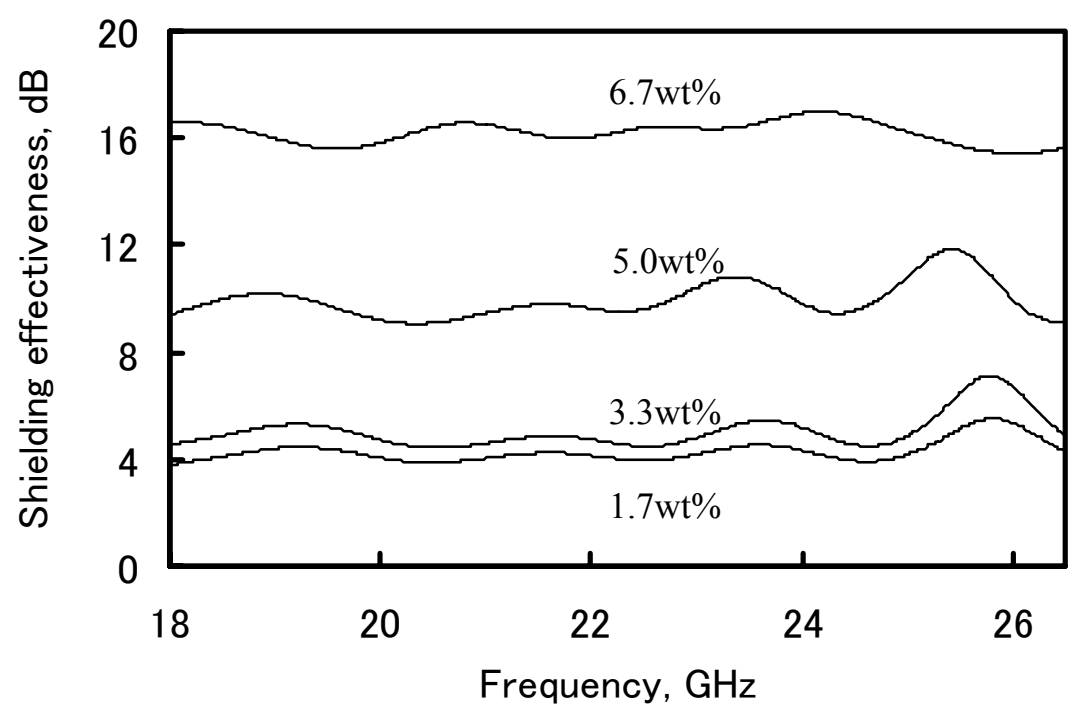

(b)

Fig.7 The shielding effectiveness in K-band frequency range for CNT/SMP nanocomposites with different thick (a) $3 \mathrm{~mm}$, (b) $0.5 \mathrm{~mm}$ 


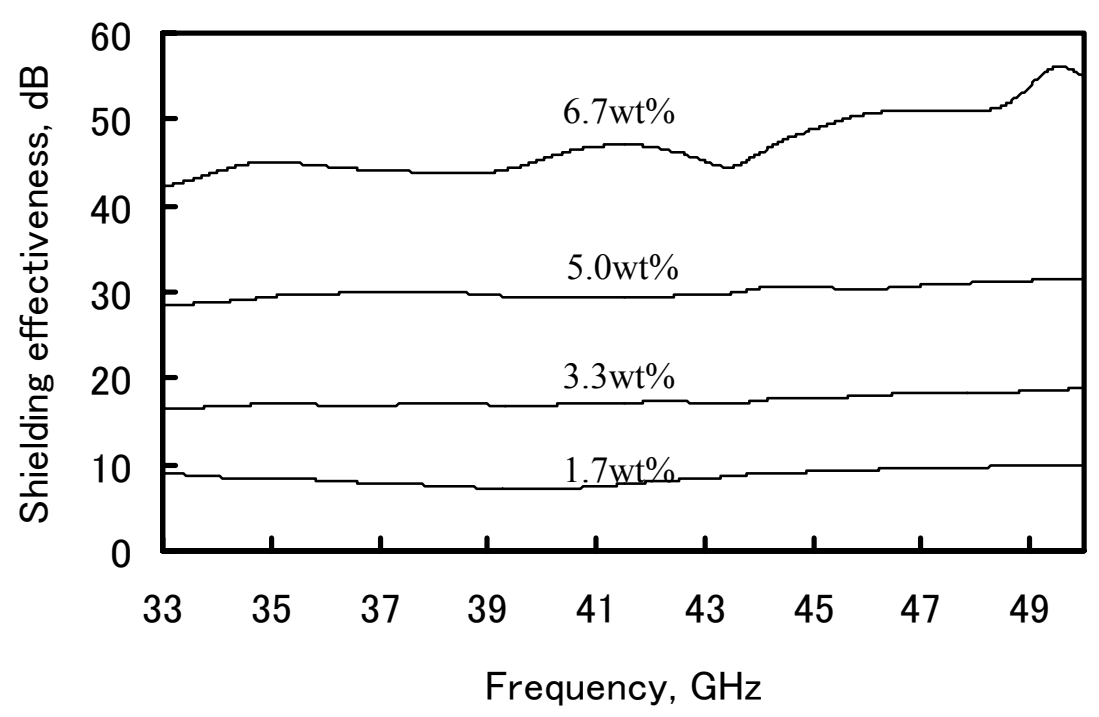

(a)

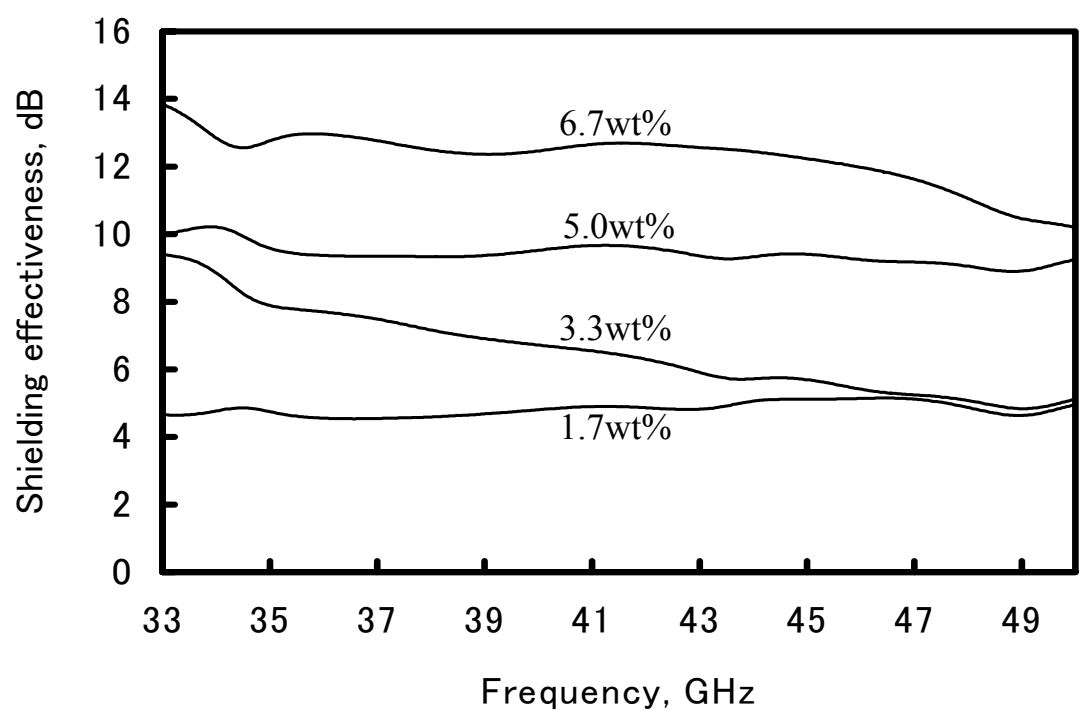

(b)

Fig.8 The shielding effectiveness in Q-band frequency range for CNT/SMP nanocomposites with different thick (a) $3 \mathrm{~mm}$, (b) $0.5 \mathrm{~mm}$ 


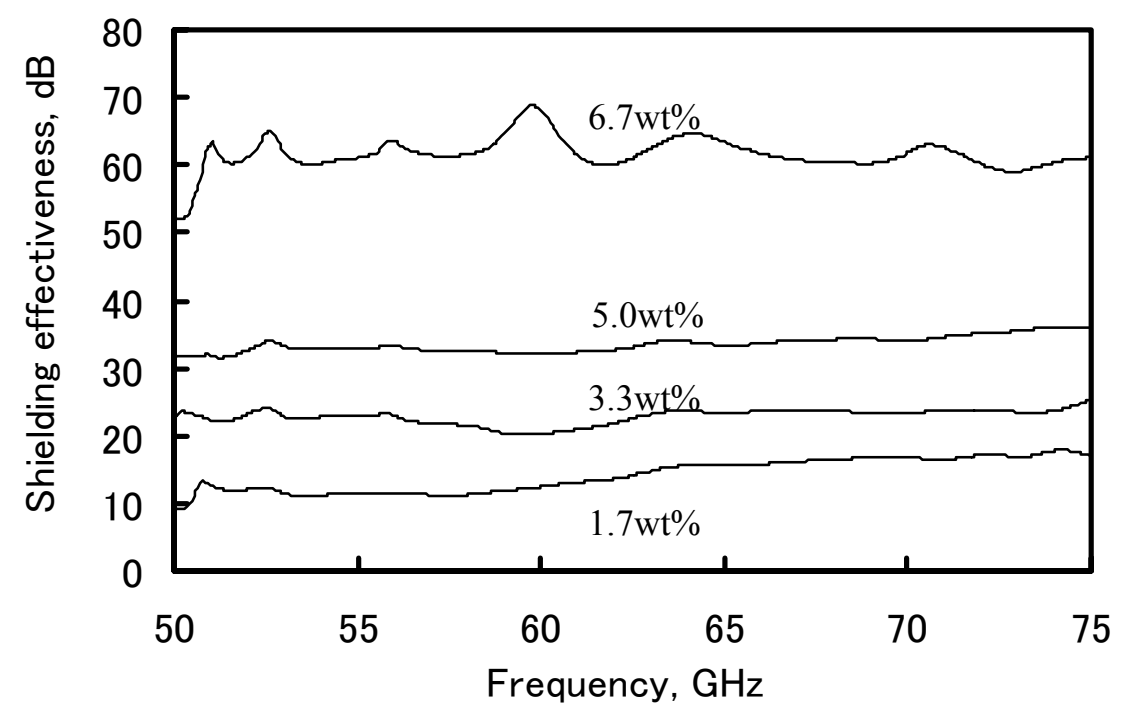

(a)

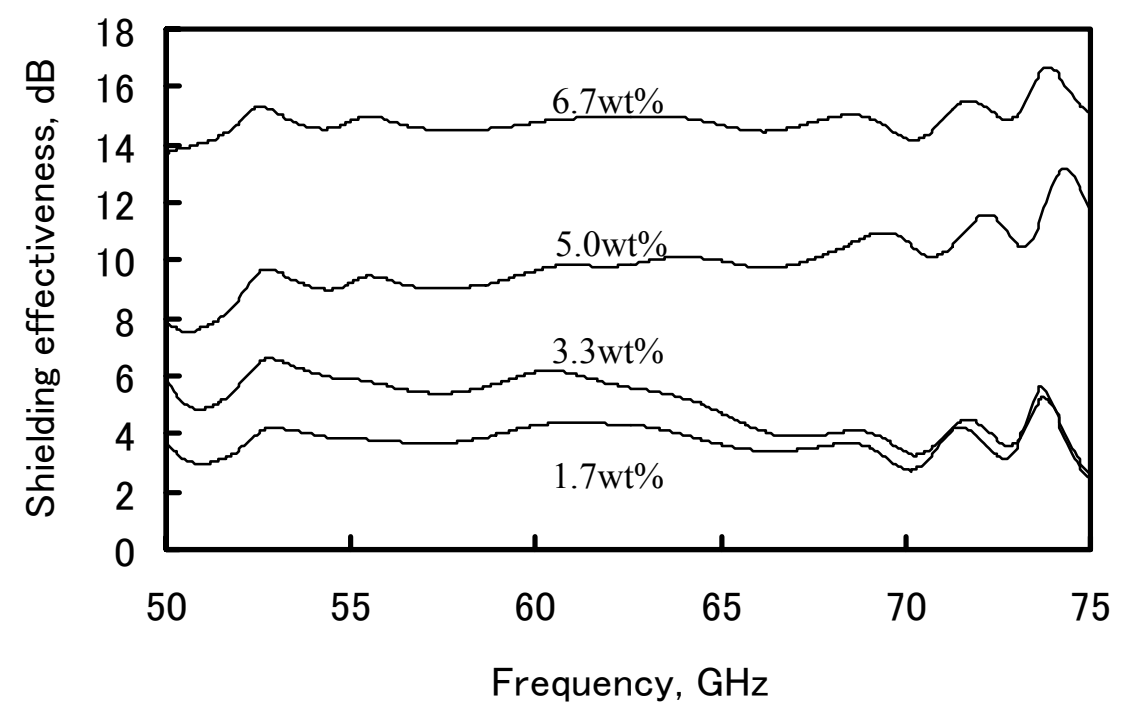

(b)

Fig.9 The shielding effectiveness in V-band frequency range for CNT/SMP nanocomposites with different thickness (a) $3 \mathrm{~mm}$, (b) $0.5 \mathrm{~mm}$ 
(a)
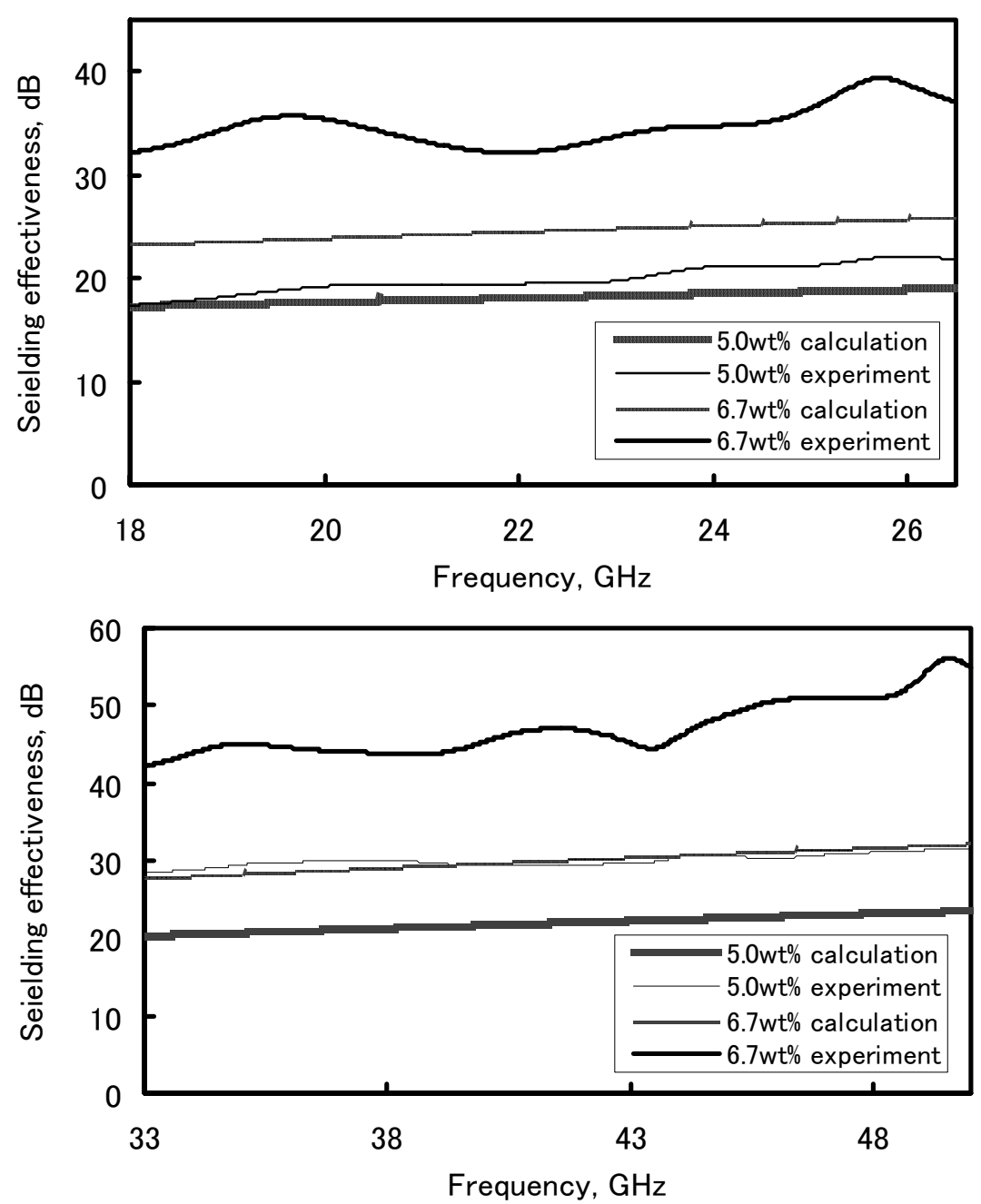

(c)

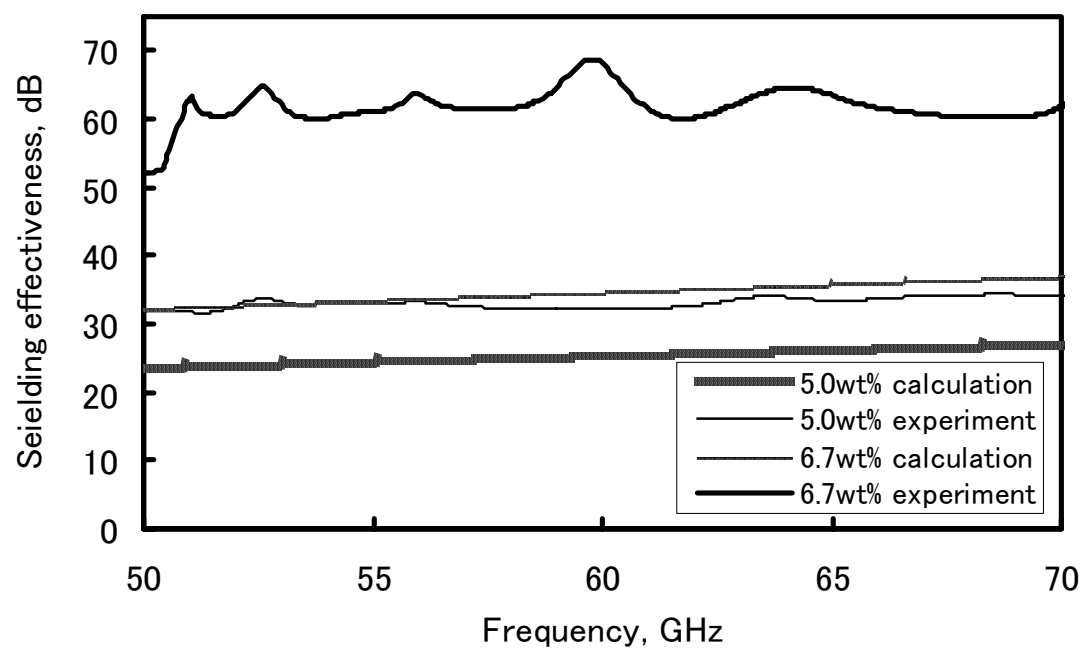

Fig. 10 The shielding effectiveness for CNT/SMP nanocomposites at thickness $3 \mathrm{~mm}$ with different frequency range by theoretical calculation (a) K-band, (b) Q-band, (c) V-band 